\title{
A case of infratemporal fossa Aspergilloma presenting as
}

\section{otitis externa}

C Y Y Wong, R Gan, S Ghosh, L Condon.

Department of Otolaryngology, Pinderfields General Hospital, Wakefield

WHS

The Mid Yorkshire Hospitals

\section{Background}

- We report a case to highlight the difficulties faced in making a precise diagnosis in an extensive infratemporal fossa Aspergilloma presenting as non-resolving otitis externa to empirical antimicrobial treatment

- In this case bacterial and fungal swabs and cultures failed to provide a diagnosis

- Antifungal treatment (Liposomal Amphotericin B followed by Voriconazole) led to significant clinical improvement, reflecting on subsequent repeat imaging with reversal of bone destruction

\section{Case Presentation}

- 67 year-old gentleman with a background of well controlled type 2 diabetes mellitus.

- Two-month history Left sided Otalgia, pre-auricular region radiating to the jaw and temple, treated for otitis externa in but failed to improve.

- Examination: white debris in external acoustic canal (EAC), inflamed deep canal wall obstructing view of the tympanic membrane, intact facial nerve

- Symptoms improved within a month following surgery, but residual pain surrounding TMJ. In subsequent months developed swelling over his left TMJ and parotid and a grade 4 facial palsy

- Patients' pre-auricular pain and swelling improved over the course of 2 months of IV AmBisome, regular ear toileting and topical antibiotic ear drops. Unfortunately, there was no improvement of facial palsy and now currently has an almost complete left sided hearing loss

\section{Investigations}

- Audiometry : mild sensorineural loss on the right and a moderate mixed loss on the left

- Initial ear swabs were negative for bacteria or fungus and biopsy of the canal wall was in keeping with inflammation

- CT scan prior to surgery of the petrous temporal bones revealed thickening of the EAC with small focal erosions of the bony canal

- Biopsies of the ear canal and mastoid cavities showed granulation polypoidal tissue in keeping with inflammatory disease

- MRI following surgery : extensive soft tissue inflammation and infection with pockets of pus around the TMJ, muscles of mastication, parotid salivary glands with progressive skull base changes

- Aspergillus found in a mastoid bone marrow trabeculae following histological staining, five months following first biopsy

\section{Management}

- Initial Management : PO Ciprofloxacin + topical Betnovate $\mathrm{N}+$ topical Clotrimazole + microsuctioning for 3 weeks as outpatient

- 7 day course of oral prednisolone and empirical IV meropenem and clindamycin for 10 days during admission

- Underwent a cortical mastoidectomy and tympanotomy after symptoms persisting. IV Co-amoxiclav post-op 5 days.

- Further exploration of the left ear and TMJ Multiple tissue biopsies from anterior canal wall, TMJ, temporal fascia, pre-auricular and parotid were obtained for histopathological analysis

- Joint decision between OMFS, otolaryngology and microbiology to prioritize the commencement of a long course of intravenous antifungal Amphotericin B (AmBisome) following this with further cultures and sensitivities.
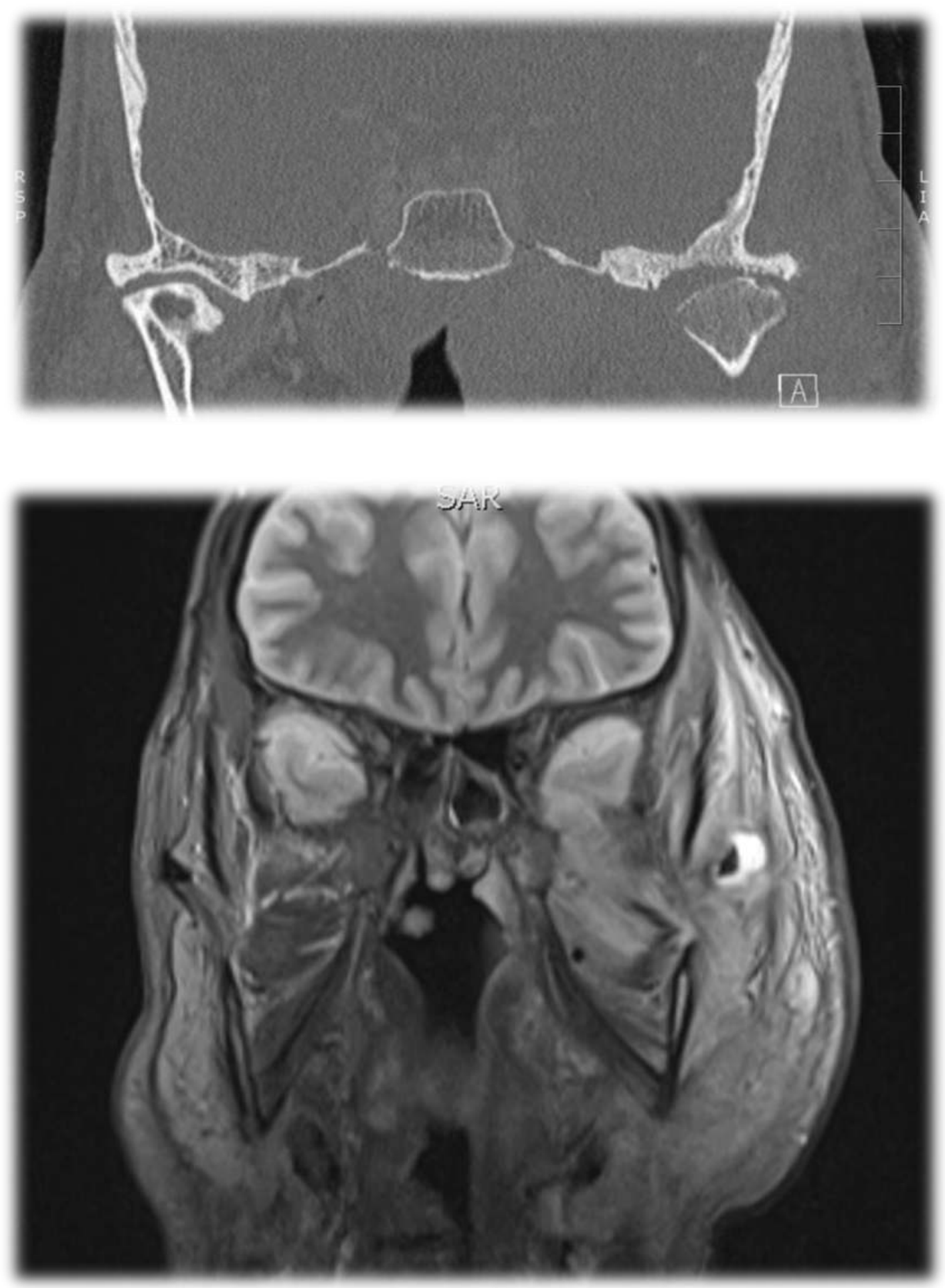

\section{Conclusions}

- Non-resolving otitis externa should prompt further search in regions such as the infratemporal fossa and skull base and a wider search for causative pathogens.

- Aspergillus may be identified on microscopy however it can be mistaken for other filamentous fungi with similar structures and thus microscopy alone is usually unable to distinguish between the moulds [8]. Histological examination of specimens can help but again the appearances of the different moulds can look similar [8]. Aspergillus culture grows quickly within 3 days but species identification requires sporulation [9]. Some fungus species have slow-sporulation [9].

- Difficulty in establishing fungal aetiology should support early initiation of antifungal treatment.

References
$[1]$ "Soudry, Hamzany Y, Preis M, Joshua B, Hadar T, Nageris Bl. Malignant external otitis: analysis of severe cases. Otolaryngology-Head and Neck Surgery. 2011 May; 144(5):758-62."

[2] "Li W, Qiao X, Yang L. Chronic invasive fungal rhinosinusitis complicated with infratemporal fossa fungal infection: 2 cases report. Lin chuang er bi yan hou tou jing wai ke za zhi= Journal of clinical otorhinolaryngology, head, and neck surgery. 2014 Feb;28(4):276-9.".

[3] "Lee DH, Kim SK, Joo YE, Lim SC. Fungus ball within a mucocele of the sphenoid sinus and infratemporal fossa: case report with radiological findings. The Journal of Laryngology \& Otology. 2012 Feb; 126(2):210-3.".

imaging findings. Neuroradiology. 2013 Apr 1;55(4):467-73.".

[5] "Waizel-Haiat S, Cohn-Zurita F, Vargas-Aguayo AM, Ramírez-Aceves R, Vivar-Acevedo E. Chronic invasive rhinocerebral mucormycosis. Cirugia y cirujanos. 2003; 71(2):145-9.".

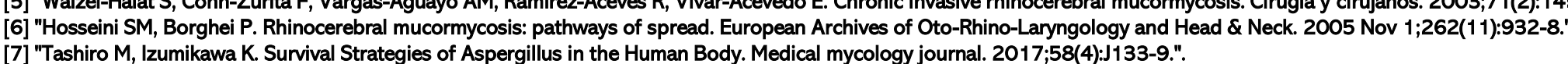

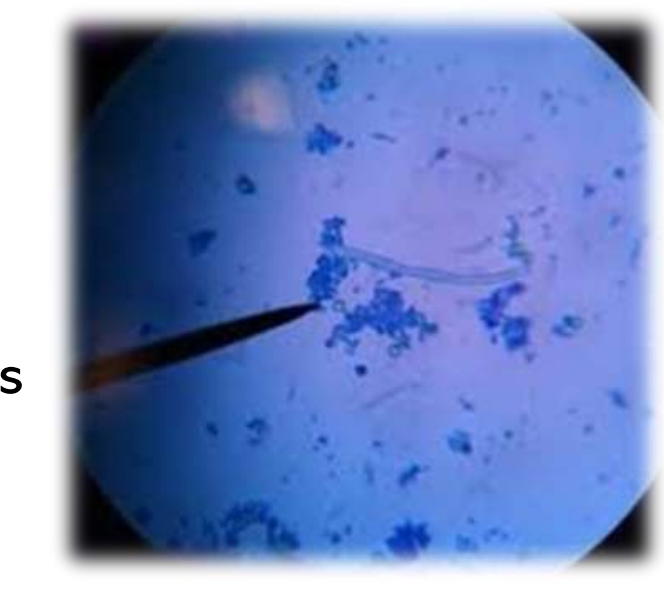

